\title{
On the influence of emotion on conflict processing
}

\author{
Philipp Kanske* \\ Department of Social Neuroscience, Max Planck Institute for Human Cognitive and Brain Sciences, Leipzig, Germany \\ ${ }^{*}$ Correspondence: kanske@cbs.mpg.de
}

Edited by:

Mara Mather, University of Southern California, USA

Reviewed by:

Kristin Prehn, Freie Universität Berlin, Germany

Noga Cohen, Ben-Gurion University of the Negev, Israel

\section{INTRODUCTION}

The ability to show coherent goal-directed behavior, even in the presence of distraction, requires the detection and resolution of conflict, for example between opposing action tendencies. This defining feature of cognitive control has been extensively studied with experimental tasks like the Stroop, Simon, or Flanker paradigm (Stroop, 1935; Simon and Rudell, 1967; Eriksen and Eriksen, 1974), which also yielded the description of an underlying neural network for conflict detection and resolution (Fan et al., 2003; Ridderinkhof et al., 2004). Understanding the influence of emotion on this system is particularly important because they are intrinsically tied to one another in real life (Pessoa, 2008). Recent evidence seems to be contradictory with reports of facilitation and impairment effects of emotion on the processing of conflict. On a conceptual basis both accounts can be supported. It may be argued that keeping up goal-directed behavior is especially difficult in emotional situations because of their greater distracting potential. On the other hand, emotional stimuli are also signals of relevance for a situation that might require particularly efficient cognitive control (Scherer, 1994). In line with this latter view, Norman and Shallice (1986) suggested that emotion, along with other instances like novelty or error commitment, might trigger cognitive control processes. The present opinion article aims at elucidating these diverging views by specifying two critical factors - task relevance and individual differences - that result in an enhancing or hindering influence of emotion on the processing of conflict.

\section{TASK RELEVANCE}

To study conflict processing, participants are typically given a certain goal related to a target stimulus and need to ignore other irrelevant information. The irrelevant information could be related to peripheral distractor stimuli (e.g., in the Flanker task) or to stimulus-response-associations (e.g., in the Simon task). If this information is incongruent with the given goal the resulting conflict needs to be detected and solved in order to show coherent behavior. A series of recent studies demonstrated that the speed, with which conflict is resolved in such tasks is enhanced, if the presented conflict stimuli are emotional, i.e., emotion speeds up cognitive conflict processing (Kanske and Kotz, 2010, 2011b). This effect is independent of emotional valence and observable for positive and negative stimuli alike (Kanske and Kotz, 2011a). It is also present in the visual and auditory modality (Kanske and Kotz, 2011d) and in Flanker, as well as Simon-type conflict tasks (Kanske and Kotz, 2011b,c). The latter is especially interesting as the mechanisms underlying Flanker and Simon tasks are very different. Conflict in the Simon task is not elicited through peripheral distractor stimuli, but through incompatible stimulus presentation and response side mapping. If, for example participants perform a voice gender discrimination with female voices requiring a right hand response, but the stimulus is presented on the left side, the elicited action tendencies will conflict and responses are prolonged. Even though this task does not require participants to focus on emotions, responses in the incompatible condition are accelerated if the spoken word is emotional (Kanske and Kotz, 2011b). The presence of the emotional modulation of conflict processing across these different conflict tasks and different sensory modalities strongly suggests that it is a general mechanism acting on the supra-modal processing steps of conflict detection and resolution rather than on visual or auditory spatial attention. Critically, however, in each of these studies the emotionally valent stimuli were task-relevant, i.e., the stimuli that participants needed to process and react to in order to solve the task. This contrasts other studies that modulated emotion through the presentation of additional stimuli that were unrelated to the task. For example, when emotional stimuli are presented before the conflict stimuli, conflict resolution is not facilitated, but impaired (Hart et al., 2010). Furthermore, conflict adaptation, i.e., the enhanced processing of conflict after presentation of an incongruent trial, is also compromised if emotional stimuli are presented between conflict trials (Padmala et al., 2011). This pattern of hindering and enhancing effects of emotion on conflict processing suggests that the exact role that emotion plays in a task is crucial for determining its impact. Emotion will only enhance cognitive control if the behaviorally relevant stimuli that participants react to during a task are emotional. Transiently induced emotion by task-unrelated stimuli, in contrast, yields hindering effects (for potentially conflicting results see Birk et al., 2011; Melcher et al., 2012, but see also Cohen and Henik, 2012, in this Research Topic for a discussion). Corroborating evidence for this conclusion also comes from two recent studies on the effects of motivation on conflict processing. Similarly to emotional stimuli that signal importance of a situation, if relevance is increased through reward for correct task performance, cognitive control is triggered resulting in accelerated conflict processing (Padmala and Pessoa, 2011). If, however, the motivational salience of the distractor stimuli is increased, conflict resolution is impaired (Krebs et al., 2010). It may thus be relevance in general that speeds up conflict processing, rather than emotion specifically. 


\section{NEURAL UNDERPINNINGS}

A crucial node in the neural network underlying cognitive control is the anterior cingulate cortex (ACC), in particular its dorsal portion (Ridderinkhof et al., 2004). The ventral ACC has been shown to be more sensitive to emotion (Bush et al., 2000) and also to conflict between emotional stimuli of different valence (Etkin et al., 2006). This ventral portion is also heavily connected to the amygdala, which is critically involved in emotion detection and generation (Phelps and LeDoux, 2005). In the context of emotional influences on conflict processing, interactions between these three regions seem important. Conflicting stimuli that are emotionally negative activate the ventral ACC in addition to the dorsal portion, which is active for incongruent stimuli irrespective of their emotional status (Kanske and Kotz, 2011b). Furthermore, functional connectivity between the ventral and dorsal ACC, as well as between ventral ACC and the amygdala is increased in incongruent emotional compared to neutral trials (Kanske and Kotz, 2011c). This suggests that the ACC is integrating the need for increased cognitive control in conflicting situations with task-relevant emotional stimuli. The emotional saliency information is signaled by the amygdala and the ACC prioritizes these situations resulting in enhanced conflict processing. The relative speed of this process is apparent in an emotional modulation of the first conflict-related component of the event-related potential of the EEG (Kanske and Kotz, 2011a). Already 200 ms after stimulus onset, emotional incongruent stimuli elicit an enhanced negativity over central electrodes, which has been localized to the dorsal ACC in non-emotional conflict processing (N200; van Veen and Carter, 2002). The effects of task-irrelevant emotional stimuli on reducing conflict processing efficiency are also mediated by the ACC. Activation reduction in this region during emotional distraction correlates with increased response times in the conflict task (Hart et al., 2010). The data on the neural underpinnings of reward-related influences on conflict processing show some similarities with the nucleus accumbens involved in behavioral facilitation of conflict resolution by reward associated stimuli, and a medial frontal region just dorsal to the ACC integrating information on the reward value of distractor stimuli (Krebs et al., 2011).

\section{INDIVIDUAL DIFFERENCES}

As individuals differ in sensitivity to emotional stimuli and cognitive control capabilities, there are also variations in the modulation of conflict processing through emotion. A recent correlational study examined this across six experiments on the influence of task-relevant emotional stimuli on conflict processing (Kanske and Kotz, 2012). Individuals high in subclinical depression and anxiety, both emotional states with deficient emotion processing (Kalia, 2005; Li et al., 2008), show a reduced increase in cognitive control for negative emotional stimuli. In contrast, the temperament trait effortful control, which correlates positively with conflict processing and describes the ability for self-regulation (Gerardi-Caulton, 2000) is associated with an enhanced emotion induced facilitation of conflict processing. These relations also translate to the neural level. High depression and anxiety are characterized by a smaller conflict-related increase in ventral ACC activity and N200 amplitude in taskrelevant emotional stimuli, while effortful control has the opposite effects. Anxiety might also be related to the impairing effect of task-irrelevant emotional stimuli on conflict processing, but the evidence regarding the direction of this effect is rather mixed (Dennis and Chen, 2007; Dennis et al., 2008). The presence of these individual differences and their systematic relation to emotional states and temperament across different experimental designs (Kanske and Kotz, 2012) may therefore represent an important aspect when explaining the enhancing and hindering effects of emotion on cognitive control.

\section{INTEGRATION}

The discussed results suggest a tight integration of emotion and cognitive control in situations that require the resolution of conflict. Figure 1 illustrates this for conflict processing in neutral stimuli (Figure 1A), and for an emotional task-irrelevant (Figure 1B) or task-relevant (Figure 1C) stimulus. In the well-described situation of conflict in neutral stimuli, cognitive control resources will be recruited and bias information processing in line with current task-demands through amplification of the cortical representation of the task-relevant stimulus (Egner and Hirsch, 2005). If a task-unrelated stimulus is emotional, relevance detectors like the amygdala or nucleus accumbens will divert resources toward the processing of that stimulus, thereby impairing the processing of task-related conflict (Pessoa, 2009). The ACC closely follows this pattern by showing emotion-related activation increase that predicts impaired task performance (Lim et al., 2008). ACC activity related to conflict, however, is decreased under emotional distraction, which also correlates with impaired behavior (Hart et al., 2010). In contrast, when the task-relevant stimulus is emotional, the current task-goal and emotional information signaled through the relevance detector coincide. Therefore, increased cognitive control resources will be recruited yielding an enhanced bias in information processing toward task-relevance. Here, the ACC also follows this pattern with the additional activation of the ventral portion for conflict in emotional stimuli, where the resolution of conflict is accelerated compared to neutral stimuli (Kanske and Kotz, $2011 b, c)$. These interactions are variable, for example with individual differences in emotion-related amygdala reactivity and limbic-prefrontal connectivity in depression and anxiety (Johnstone et al., 2007; Kanske and Kotz, 2012). Here, the altered relevance detection seems to result in the dysfunctional integration of emotion and current task-goals apparent in reduced ventral ACC activation. This consequently leads to impaired performance.

\section{CHALLENGES AND OUESTIONS}

The evident pattern might show some valence specificity. Even though negative and positive emotional stimuli yield comparable behavior and EEG results (Kanske and Kotz, 2010, 2011a), there is less data yet on the neural mechanisms underlying the influence of positive emotion on conflict processing. As depression and anxiety selectively affect the influence of negative, but not positive emotional stimuli on conflict processing (Kanske and Kotz, 2012), the mechanisms that mediate the influence on conflict processing might be different (see also Prehn et al., 2011). For positive emotion they might resemble more the pattern observed for reward-related stimuli with nucleus accumbens involvement (Krebs et al., 2011). The mechanisms might also differ for highly salient emotional stimuli, as suggested by Pessoa (2009). This notion is supported by the observed 

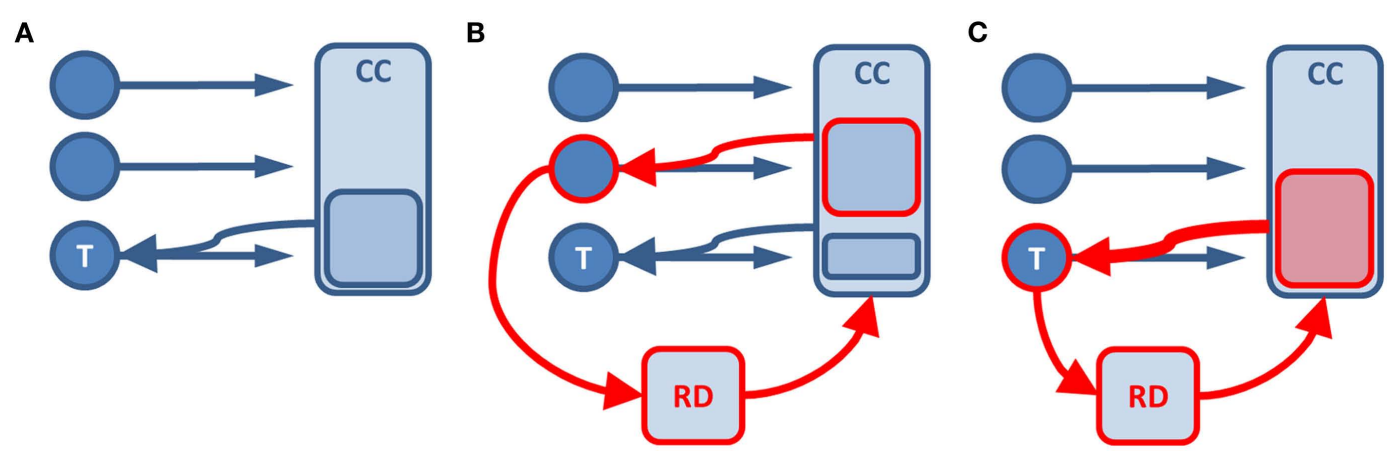

FIGURE 1 | Illustration of the functional integration of emotion and cognitive control in conflict processing. The situation for neutral (A) and emotional task-irrelevant (B) or task-relevant stimuli (C) is displayed. Circles represent stimuli. Rectangles stand for processing units. Red frame color indicates an emotional stimulus or emotion induced processing. T, task-relevant stimulus; CC, cognitive control; RD, relevance detector. effects for anxiety and depression, which are characterized by increased sensitivity to negative emotional stimuli and increased related amygdala responding (Kanske and Kotz, 2012). The similarity of the results for motivation and emotion suggests that it may be relevance in general that yields enhanced conflict processing. This question should be followed up by direct comparisons of differently salient stimuli. Lastly, the relation of emotion and cognitive control is not uni-directional, rather, different levels of control may also affect emotion. There is some indication that individuals with high emotion regulation capacity attenuate emotional processing when cognitive control is recruited for conflict processing (Cohen et al., in press). The challenge for future investigations will be to concurrently study these reciprocal relations and their underlying neural mechanisms.

\section{CONCLUSION}

Conflict processing is directly influenced by emotion, with individual differences in temperament and emotional state, as well as the task-relevance of the emotionally valent stimuli critically determining if this influence is an enhancing or a hindering one. The resulting pattern is highly evolutionary adaptive as the time that conflict yields an organism incapable of responding to potentially dangerous negative or rewardsignaling positive stimuli is reduced. In contrast, if stimuli are present that are not relevant to current task-goals, but may still be relevant to higher order goals because of their danger- or reward-value, their processing is prioritized over task-performance. In consequence, the flexibility of behavior is greatly enhanced with slower and faster processing routes depending on current task-related and overarching goals.

\section{REFERENCES}

Birk, J.L., Dennis, T.A., Shin, L. M., and Urry, H.L. (2011). Threat facilitates subsequent executive control during anxious mood. Emotion 11, 1291-1304.

Bush, G., Luu, P., and Posner, M. I. (2000). Cognitive and emotional influences in anterior cingulate cortex. Trends Cogn. Sci. (Regul. Ed.) 4, 215-222.

Cohen, N., and Henik, A. (2012). Do irrelevant emotional stimuli impair or improve executive control? Front. Integr. Neurosci. 6:33. doi: 10.3389/fnint.2012.00033

Cohen, N., Henik, A., and Moyal, N. (in press). Executive control attenuates emotional effects-for high reappraisers only? Emotion.

Dennis, T.A., and Chen, C.C. (2007). Neurophysiological mechanisms in the emotional modulation of attention: the interplay between threat sensitivity and attentional control. Biol. Psychol. 76, 1-10.

Dennis, T. A., Chen, C. C., and Mccandliss, B. D. (2008). Threat-related attentional biases: an analysis of three attention systems. Depress. Anxiety 25, E1-E10.

Egner, T., and Hirsch, J. (2005). Cognitive control mechanisms resolve conflict through cortical amplification of task-relevant information. Nat. Neurosci. 8, 1784-1790.

Eriksen, B. A., and Eriksen, C. W. (1974). Effects of noise letters upon the identification of a target letter in a nonsearch task. Percept. Psychophys. 16, 143-149.

Etkin, A., Egner, T., Peraza, D. M., Kandel, E. R., and Hirsch, J. (2006). Resolving emotional conflict: a role for the rostral anterior cingulate cortex in modulating activity in the amygdala. Neuron 51, 871-882.

Fan, J., Flombaum, J. I., Mccandliss, B. D., Thomas, K. M., and Posner, M. I. (2003). Cognitive and brain consequences of conflict. Neuroimage 18, 42-57.

Gerardi-Caulton, G. (2000). Sensitivity to spatial conflict and the development of self-regulation in children 24-36 months of age. Dev. Sci. 3, 397-404.

Hart, S. J., Green, S. R., Casp, M., and Belger, A. (2010). Emotional priming effects during Stroop task performance. Neuroimage 49, 2662-2670.

Johnstone, T., van Reekum, C. M., Urry, H. L., Kalin, N. H., and Davidson, R. J. (2007). Failure to regulate: counterproductive recruitment of top-down prefrontal-subcortical circuitry in major depression. J. Neurosci. 27, 8877-8884.

Kalia, M. (2005). Neurobiological basis of depression: an update. Metab. Clin. Exp. 54, 24-27.

Kanske, P., and Kotz, S. A. (2010). Modulation of early conflict processing: N200 responses to emotional words in a flanker task. Neuropsychologia 48 , 3661-3664.

Kanske, P., and Kotz, S. A. (2011a). Conflict processing is modulated by positive emotion: ERP data from a flanker task. Behav. Brain Res. 219, 382-386.

Kanske, P., and Kotz, S. A. (2011b). Emotion speeds up conflict resolution: a new role for the ventral anterior cingulate cortex? Cereb. Cortex 21, 911-919.

Kanske, P., and Kotz, S. A. (2011c). Emotion triggers executive attention: anterior cingulate cortex and amygdala responses to emotional words in a conflict task. Hum. Brain Mapp. 32, 198-208.

Kanske, P., and Kotz, S. A. (2011d). Positive emotion speeds up conflict processing: ERP responses in an auditory Simon task. Biol. Psychol. 87, 122-127.

Kanske, P., and Kotz, S. A. (2012). Effortful control, depression, and anxiety correlate with the influence of emotion on executive attentional control. Biol. Psychol. 9, 88-95.

Krebs, R. M., Boehler, C. N., Egner, T., and Woldorff, M. G. (2011). The neural underpinnings of how reward associations can both guide and misguide attention. J. Neurosci. 31, 9752-9759.

Krebs, R. M., Boehler, C. N., and Woldorff, M. G. (2010). The influence of reward associations on conflict processing in the Stroop task. Cognition 117, 341-347.

Li, W., Paller, K. A., and Zinbarg, R. E. (2008). Conscious intrusion of threat information via unconscious priming in anxiety. Cogn. Emot. 22, 44-62.

Lim, S. L., Padmala, S., and Pessoa, L. (2008). Affective learning modulates spatial competition during lowload attentional conditions. Neuropsychologia 46, $1267-1278$.

Melcher, T., Obst, K., Mann, A., Paulus, C., and Gruber, O. (2012). Antagonistic modulatory influences of negative affect on cognitive control: reduced and enhanced interference resolution capability after the induction of fear and sadness. Acta Psychol. (Amst.) 139, 507-514.

Norman, D.A., and Shallice, T. (1986). Attention to Action: Willed and Automatic Control of Behavior. New York: Plenum Press. 
Padmala, S., Bauer, A., and Pessoa, L. (2011). Negative emotion impairs conflict-driven executive control. Front. Psychol. 2: 192. doi: 10.3389/ fpsyg.2011.00192

Padmala, S., and Pessoa, L. (2011). Reward reduces conflict by enhancing attentional control and biasing visual cortical processing. J. Cogn. Neurosci. 23, 3419-3432.

Pessoa, L. (2008). On the relationship between emotion and cognition. Nat. Rev. Neurosci. 9, 148-158.

Pessoa, L. (2009). How do emotion and motivation direct executive control? Trends Cogn. Sci. (Regul. Ed.) 13, $160-166$.

Phelps, E. A., and LeDoux, J. E. (2005). Contributions of the amygdala to emotion processing: from animal models to human behavior. Neuron 48 , 175-187.
Prehn, K., Heekeren, H. R., and van der Meer, E. (2011). Influence of affective significance on different levels of processing using pupil dilation in an analogical reasoning task. Int. J. Psychophysiol. 79, 236-243.

Ridderinkhof, K. R., Ullsperger, M., Crone, E. A., and Nieuwenhuis, S. (2004). The role of the medial frontal cortex in cognitive control. Science 306, 443-447.

Scherer, K. R. (1994). "Emotion serves to decouple stimulus and response," in The Nature of Emotion: Fundamental Questions, eds P. Ekman and R. J. Davidson (New York: Oxford University Press), 127-130.

Simon, J. R., and Rudell, A. P. (1967). Auditory S-R compatibility: the effect of an irrelevant cue on information processing. J. Appl. Psychol. 51, 300-304.
Stroop, J. (1935). Studies of interference in serial verbal reactions. J. Exp. Psychol. 18, 643-662.

van Veen, V., and Carter, C. S. (2002). The anterior cingulate as a conflict monitor: fMRI and ERP studies. Physiol. Behav. 77, 477-482.

Received: 30 April 2012; accepted: 20 June 2012; published online: 09 July 2012.

Citation: Kanske P (2012). On the influence of emotion on conflict processing. Front. Integr. Neurosci. 6:42. doi: 10.3389/fnint.2012.00042

Copyright (c) 2012 Kanske. This is an open-access article distributed under the terms of the Creative Commons Attribution License, which permits use, distribution and reproduction in other forums, provided the original authors and source are credited and subject to any copyright notices concerning any third-party graphics etc. 